\title{
Di-2-pyridylketone 4, 4-dimethyl-3-thiosemicarbazone effectively induces human colorectal carcinoma cell apoptosis via mTOR pathway
}

\author{
Qianqian $\mathrm{Fu}^{\mathrm{a}, \#}$, Shengli Wang ${ }^{\mathrm{a}, \#}$, Chuyun $\mathrm{Zhu}^{\mathrm{a}, \#}$, Zhenlong Zhou ${ }^{\mathrm{b}}$, Xiaojuan $\mathrm{Yu}^{\mathrm{c}}$, Yong Zhang ${ }^{\mathrm{d}, *}$ \\ ${ }^{a}$ The First Affiliated Hospital, Biomedical Translational Research Institute and School of Pharmacy and, and Guangdong \\ Province Key Laboratory of Molecular Immunology and Antibody Engineering, Jinan University, Guangzhou, 510632, \\ China. \\ ${ }^{b}$ Department of Biomedicine, Jinan University, Guangzhou, 510632, China. \\ 'Department of Oncology The Affiliated Huaian No.1 People's Hospital, Nanjing Medical University, Jiangsu Province
} 223300, China.

${ }^{d}$ Department of Orthopaedics, Tongji Hospital, Tongji Medical College, Huazhong University of Science and Technology, Wuhan 430030, China.

\begin{abstract}
Background: To investigate the anticancer mechanisms of di-2-pyridylketone 4,4-dimethyl-3-thiosemicarbazone (Dp44mT) in human colon cancer cells. Human colorectal carcinoma (HCC) is one of the most commonly diagnosed cancers in both males and females. Current studies have found that iron chelators can be used as novel anticancer drugs; however, the anticancer activity of iron chelators and their target genes in HCC has been rarely reported.

Methods: Dp44mT was used to treat two colorectal tumor cell lines, SW480 and HT-29. The proapoptotic effects of different concentrations of Dp44mt were measured using flow cytometry and Hoechst 33258 staining. Ferric ammonium citrate (FAC) was used as an additional iron donor to inhibit the effects of Dp44mT. Apoptosis and DNA damage-related proteins were examined by Western blot analysis.

Results: In this study, we found that the iron chelators Dp44mT could induce the apoptosis in two colorectal tumor cell lines SW480 and HT-29, upregulate the expression level of p-histone H2A.X, and inhibit the phosphorylation level of mTOR in a dose-dependent way. Those effects could be reversed by the additional iron donor FAC.

Conclusion: These data indicate that iron depletion and/or the presence of iron can modulate the HCC apoptosis progression in vitro, which may be a potential target for future HCC therapy.

Keywords: Dp44mT, cell apoptosis, mTOR, DNA damage
\end{abstract}

\section{Introduction}

Colorectal cancer is the fourth most common cause of cancer-related deaths worldwide, and annual deaths have

\footnotetext{
\# These authors contributed equally to this work and should be considered co-first authors.

* Corresponding to: Yong Zhang

Mailing address: Department of Orrthohopaedics, Tongji Hospital, Tongji Medical College, Huazhong University of Science and Technology, Wuhan 430030, China.

Email: zhangyong2018@hust.edu.cn

Received: 02 August 2021 / Accepted: 03 September 2021
}

increased to approximately 700,000 [1]. Surgical resection and chemotherapy are the primary treatments. However, the risk of resection surgery and the side effects of chemotherapy, including hair loss and neuropathy, has urged researchers to develop a new target for colorectal cancer. A major limitation of cytotoxic chemotherapy is drug resistance caused by P-glycoprotein (Pgp) [2], which greatly restricts the effects of chemotherapy.

Iron is an indispensable trace element for cell metabolism. It plays a crucial role in deoxyribonucleic acid (DNA) synthesis, oxygen transport and electron transport, and energy metabolism pathways related to adenosine triphosphate (ATP) production. Iron-chelating agents exhibit highly efficient antitumor activities [3]. Iron-chelating 
agents have a stronger inhibitory effect on tumor cell proliferation activity [3]. Iron is necessary for tumor cells because it plays a key role in the protein activity sites, and tumor cells highly expressing TRF13, iron-containing enzymes, and ribonucleic acid reductase (RR), which are involved in DNA synthesis [4]. As a potential anticancer agent, iron chelators can be combined with other anticancer drugs to enhance their anticancer activity.

As a novel class of anti-tumor agents, the iron chelator, di-2-pyridylketone 4,4-dimethyl-3-thiosemicarbazone (Dp44mT) has recently been extensively studied [5]. The antitumor mechanism of Dp44mT is related to its ability to bind, to deplete cellular iron, and to generatecytotoxic radicals [6]. In addition, Dp44mT demonstrates potent and selective anti-tumor activity [6-8], and can overcome the Pgp-related multidrug resistance by directly utilizing lysosomal Pgp-transport activity [9, 10]. This drug can significantly inhibit tumor growth and metastasis and can overcome resistance to currently used chemotherapy drugs; thus, it can be usedin clinical treatment [11]. Dp44mT induces cancer cell apoptosis by increasing the expressionof the apoptotic proteins Caspase-3 8 and 9, Bax protein and cleaved PARP in different cancer cells $[12,13]$.

Notably, Dp44mT leads to the induction of various of apoptotic markers, such as cleaved caspase 3, caspase 4, cleaved PARP and so on, in different cancer cell types $[12,13]$. However, the antitumor effects of Dp44mT on colorectal tumor cells have not yet been examined. In this research, we investigated the proapoptotic effects of Dp44mT on different kinds of colorectal tumor cell lines. Dp44mT induces colorectal tumor cell apoptosis in a dose-dependent manner, and the antagonist of Dp44mT, ferric ammonium citrate (FAC), could reverse those effects. These results indicated that cellular iron might be a possible target for colorectal tumor treatment, and Dp$44 \mathrm{mT}$ might be a potential therapy.

\section{Methods}

\section{Reagents}

Dp44mT was synthesized and characterized using standard procedures [14]. Dp44mT was dissolved in DMSO as storage concentration of $10 \mathrm{mM}$. Stock solutions of 100 mg/mL FAC (Sigma-Aldrich, St Louis, MO, USA) were prepared in deionized water. Both solutions were stored at $-20^{\circ} \mathrm{C}$ and diluted to the proper concentrations before use. In this experiment, the highest level of DMSO in media was $0.05 \%(\mathrm{v} / \mathrm{v})$.

\section{Cell Culture}

The human colon carcinoma cell lines HT29 and SW480 were obtained from the American Type Culture Collection (Manassas, VA, USA). Cells were cultured in 10\% FBSsupplemented RPMI medium with L-glutamine and maintained at $37^{\circ} \mathrm{C}$ with $5 \% \mathrm{CO} 2$.

\section{Flow cytometry}

Apoptosis of HT29 and SW480 cells was detected by flow cytometry with annexin V-FITC/PI double staining. Approximately 2 x 106 HT29 and SW480 cells were collected, washed twice with cold PBS, and added with binding buffer with annexin V-FITC/PI (10 mM Hepes/NaOH at $\mathrm{pH} 7.4,0.14 \mathrm{M} \mathrm{NaCl}, 2.5 \mathrm{mM} \mathrm{CaCl}$ ). Finally, the cells were and incubated at room temperature and under dark conditions for 15 min. Flow cytometry was used to detect cell apoptosis. Annexin V-FITC/PI double staining showed double Yang in late apoptotic or necrotic cells [15].

\section{Hoechst 33258 staining}

HT29 and SW480 cells were collected fixed with 70\% ethanol for $30 \mathrm{~s}$, and washed with PBS three times. Approximately $2 \mu \mathrm{g} / \mathrm{mL}$ Hoechst 33258 dye solution was then added , and the cells were incubated at room temperature for 30 minutes. Finally, the cells were observed under a fluorescence microscope.

\section{Protein extraction and western blotting analysis}

The cells were collected and then washed with cold PBS. The cells were lysed on ice with lysate with protease inhibitor and phosphatase inhibitor, and the protein supernatant was collected after centrifugation at $16,000 \times \mathrm{g}$ for $40 \mathrm{~min}$ at $4{ }^{\circ} \mathrm{C}$. The protein concentrations were measured using standard Bradford assays, and an equal amount of the protein was collected and then transferred to a nitrocellulose membrane, which was then closed with 5\% milk and incubated with primary antibodies against p-Histones H2A.X, mTOR, p-mTOR, and GAPDH. The membrane was washed after incubation with the primary antibodies. The membrane was then incubated with horseradish peroxidase-bound secondary antibody. Finally, the protein was exposed using a ChemiDoc MP imager. All antibodies were obtained from Santa Cruz Biotech (Santa Cruz, CA, USA). The item numbers of the antibodies against pHistones H2A.X, mTOR, p-mTOR, and GAPDH were SC-517348, SC-517348, SC-293133, and SC-47724, respectively. Finally, density analysis was performed using the ChemiDoc Image Lab software (BioRad).

\section{Statistical analysis}

Data are expressed as mean \pm standard deviation (SD) of the three experiments. Experimental data are compared using one-way ANOVA. Results are considered statistically significant when $P<0.05$.

\section{Results}

\section{Dp44mT induces SW480 and HT-29 cells apoptosis}

Annexin V-FITC/PI double staining and Hoechst 33258 staining were used to evaluate the effect of Dp44mT on the apoptosis of SW480 and HT-29 cells. The results of flow cytometry showed that Dp44mT could promoted apoptosis more strongly than that in the $0 \mu \mathrm{M}$-treated group (Figures 1A and 1B). The apoptotic percentages in the different concentrations of Dp44mT-treated groups were significantly higher than those in the $0 \mu \mathrm{M}$-treated group $(P<0.05$, Figures $1 \mathrm{C}$ and $1 \mathrm{D})$. The effect of Dp44mT on the apoptosis of SW480 and HT-29 cells is dose dependent. Treatment with $10 \mu \mathrm{M}$ Dp44mT showed 
the strongest effect on the apoptosis of SW480 and HT29 cells. Therefore, $10 \mu \mathrm{M}$ Dp44mT was selected for the subsequent experiments.

After staining with Hoechst 33258, the SW480 and HT-29 cells in the $0 \mu \mathrm{M}$ Dp44mT group had normal morphology and complete round nuclei (Figure 2), whereas cells treated with Dp44mT became rare, with small nuclei, widespread bubbles, intense fluorescent spots, and pyrosis. These findings indicated chromatin concentration and the presence of apoptotic bodies. Consistent with these results of flow cytometry, Dp44mT induced SW480 and HT-29 cell apoptosis in a dose-dependent way.

\section{FAC inhibited Dp44mT-induced SW480 and HT-29 cell apoptosis}

The salvage effect of FAC on DP44MT-induced apoptosis of SW480 and HT-29 cells was detected by flow cytometry. In this experiment, $10 \mu \mathrm{M}$ Dp44mT was chosen to induce cell apoptosis, and the percentages of apoptotic cells in the untreated control group, Dp44mT group, FAC group (100 mg/ml), and Dp44mT + FAC group were compared (Figure 3 ). The results showed that co-treatment with FAC could significantly inhibit the cell apoptosis induced by Dp44mT. Similar results were observed in both SW480 and HT-29 cells.

\section{Dp44mT induced SW480 and HT-29 cells apoptosis via the mTOR pathway}

The effects of different concentrations of Dp44mT and FAC on the protein expressions of p-Histone H2A.X, mTOR, and p-mTOR in SW480 cells were investigated. As shown in Figure 4, Dp44mT induced the protein ex-
A

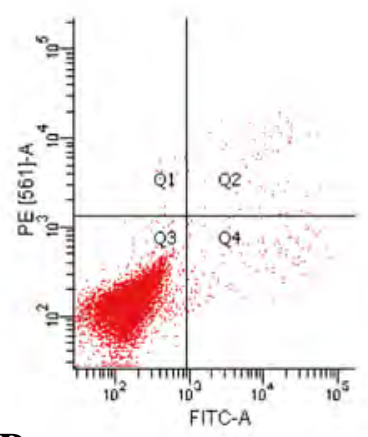

B

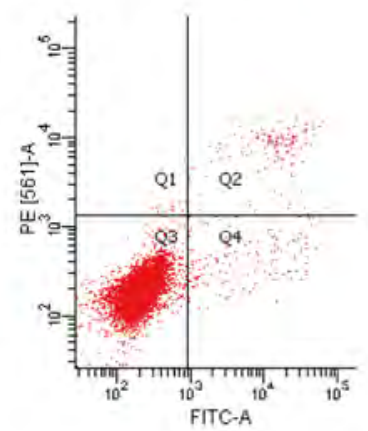

$0 \mu \mathrm{M}$ Dp44mT
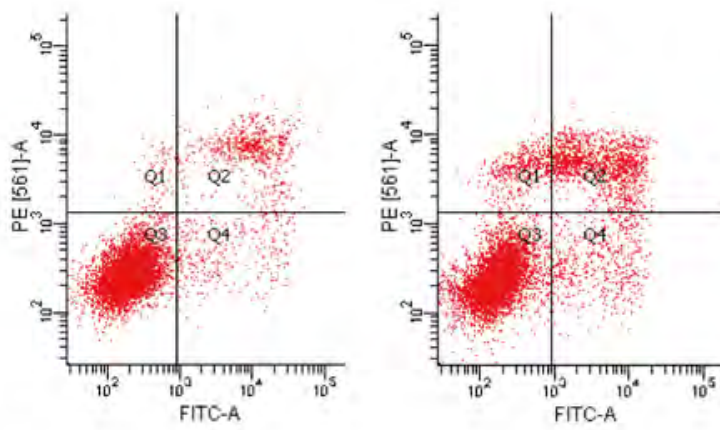

HT-29 Cell

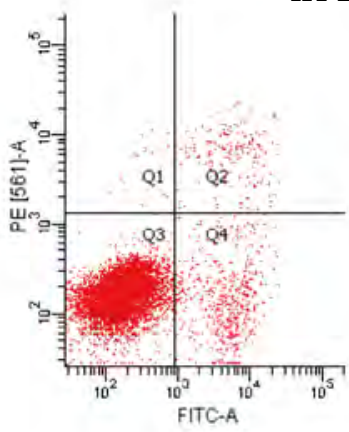

$2.5 \mu \mathrm{M}$ Dp44mT

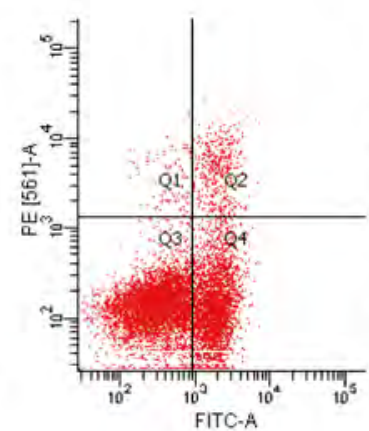

$5 \mu \mathrm{M}$ Dp44mT
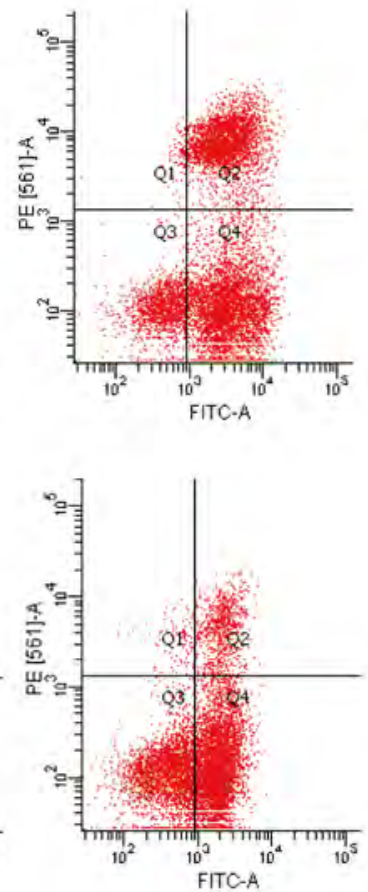

$10 \mu M$ Dp44mT
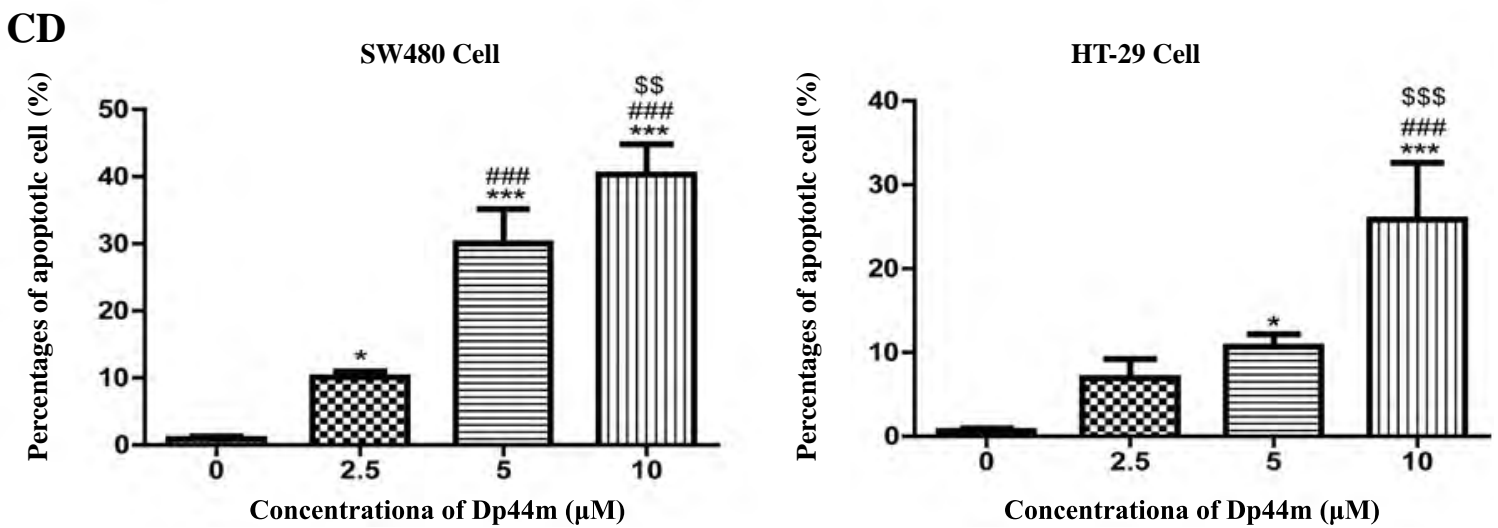

Figure 1. Dp44mT induces SW480 and HT-29 cells apoptosis. Cells were stained with annexin V-FITC/PI to verify the apoptotic cell ratio after treatment with different concentrations of Dp44mT $(0,2.5,5$, and $10 \mu \mathrm{M})$. Dp44mT induced SW480 cell (A) and HT-29 cell (B) apoptosis in a dosedependent way. Statistical results are shown in (C) and (D). ${ }^{* * * *}$ indicates $P<0.05$ and $P<0.001$ compared with the $0 \mu \mathrm{M}$ group. ${ }^{\# \#}$ means $P<0.001$ compared with the $2.5 \mu \mathrm{M}$ group. ${ }^{\$ \$}$ and ${ }^{\$ \$}$ mean $P<0.01$ and $P<0.001$ compared with the $5 \mu \mathrm{M}$ group, respectively; $n=3$. 
A

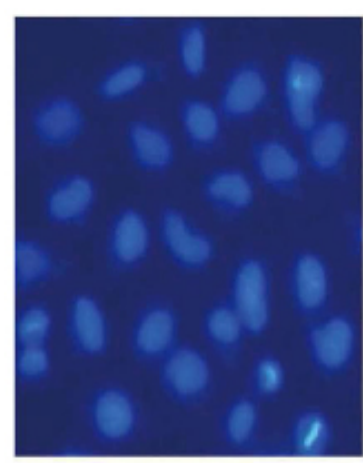

B

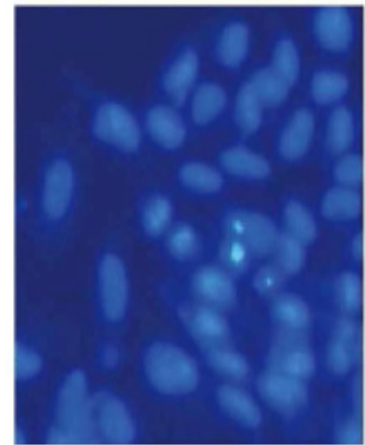

$0 \mu \mathrm{M}$ Dp44mT
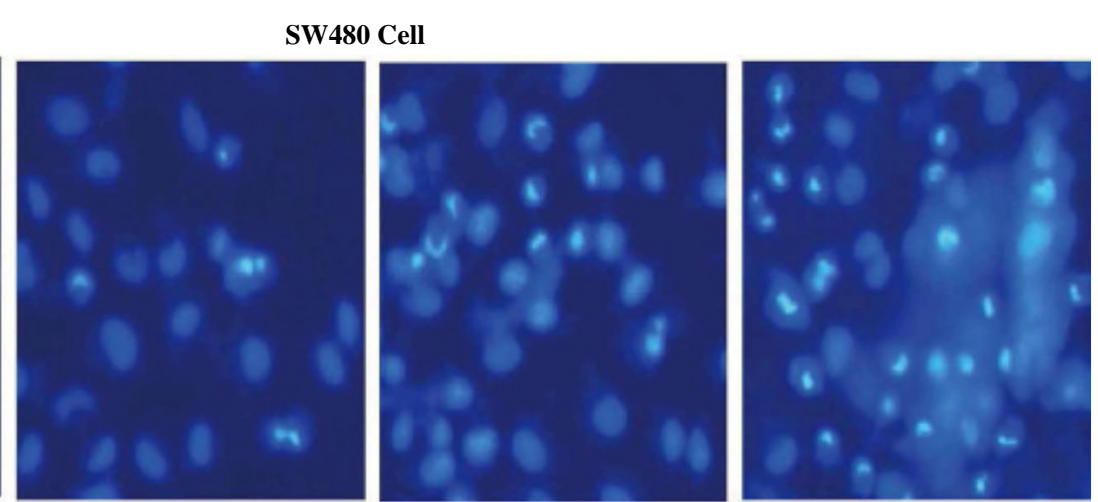

HT-29 Cell

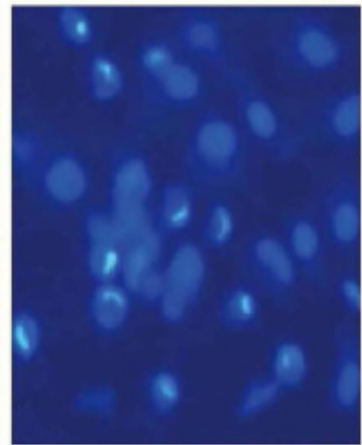

$2.5 \mu \mathrm{M}$ Dp44mT

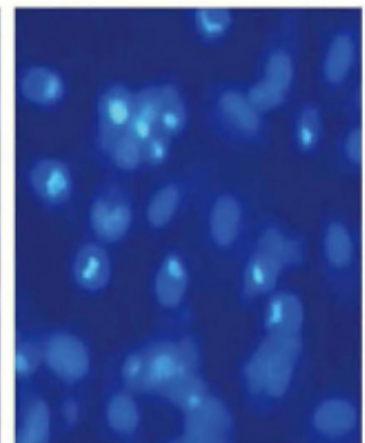

$5 \mu \mathrm{M}$ Dp44mT

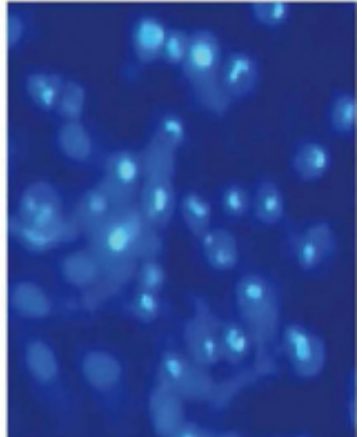

$10 \mu M$ Dp44mT

Figure 2. Dp44mT induced SW480 and HT-29 cells apoptosis. Hoechst 33258 staining indicated that Dp44mT induced SW480 (A) and HT-29 cell (B) apoptosis in a dose-dependent way. Morphological changes in nuclei observed with Hoechst 33258 staining under fluorescence microscopy. Untreated cells showed a normal shape with round intact nuclei, and the obvious morphological changes were observed in Dp44mT-treated cells. The original magnification is $400 \times$. The scale bar is $1: 400$.

pressions of p-Histone H2A.X and inhibited the phosphorylation of mTOR in a dose-dependent way. Cells treated with Dp44mT and FAC showed lower expression of p-Histone H2A.X and higher expression of mTOR and p-mTOR compared with that in the Dp44mT-treated cells. These results indicated that FAC inhibited Dp44mTinduced p-Histone H2A.X expression and recovered the phosphorylation of mTOR in SW480 cells.

\section{Discussion}

Increased drug resistance to standard treatment among cancers has lead to the investigation of new therapeutic strategies. As a result, the implication of the Dp44mT and its analogues was emerged as new anticancer therapeutics, as suggested by their broad anti-tumoractivities $[6,16,17]$ their effects on drug resistance [18] and tumor metastasis [19], and their oral bioavailability and tolerability. Di2-pyridylketone 4-cyclohexyl-4-methyl-3-thiosemicarbazon is a kind of Dp44mT analogues that has been used in entered a multi-center clinical trial to treat advanced and drug-resistant tumors in early 2016 [11].

In the present study, the effects of different concentrations of Dp44mT on colorectal cancer cell apoptosis were explored. The results indicated that Dp44mT could induce cell apoptosis in a dose-dependent way. FAC, which was usually used as the agonist of Dp44mT, could significantly inhibited Dp44mT-induced cell apoptosis. The exploration of mechanisms indicated that the proapoptotic effects of Dp44mT on colorectal cancer cells were related to the promoted expression of p-Histone H2A.X and the inhibition of mTOR and p-mTOR. The rescued effects of FAC partially contributed to its inhibition on Dp44mT-induced p-Histone H2A.X expression and recovery of phosphorylation of mTOR.

The iron-chelating agent can be used as an anticancer agent to induce the apoptosis of cancer cells mainly by activating mitochondrial apoptosis induced by the caspase pathway; therefore, the proliferation of cancer cells can be inhibited by regulating apoptosis [20]. Dp44mT is a very effective antitumor-chelating agent, and studying its effect on apoptosis is of great significance.

The ability of a chelator to bind cellular iron leads to apoptosis [20]. If iron chelators lead to tumor cell death by influencing the apoptotic pathway, then regulating apoptosis becomes important in inhibiting cancer cell proliferation. Given that $\mathrm{Dp} 44 \mathrm{mT}$ was the most effective chelator yet screened for antitumor activity, it was crucial to assess its ability to induce apoptosis.

In this study, we found that $\mathrm{Dp} 44 \mathrm{mT}$ could promote the expression of p-Histone H2A.X, indicating double-strand 
A

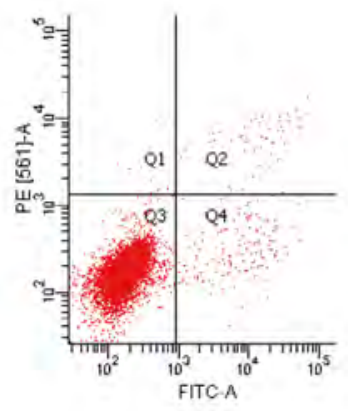

B

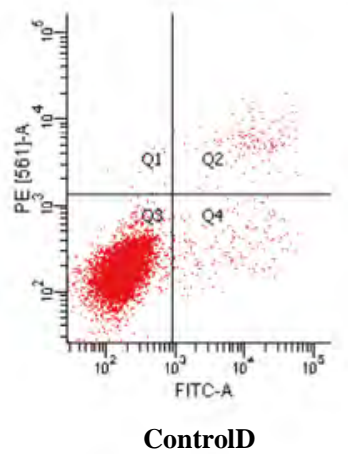

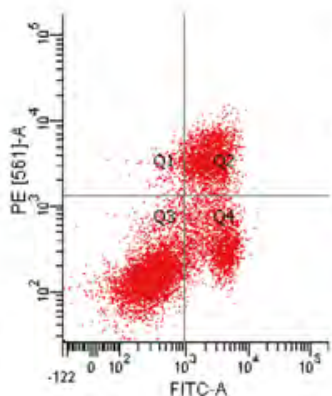

HT-29 Cell
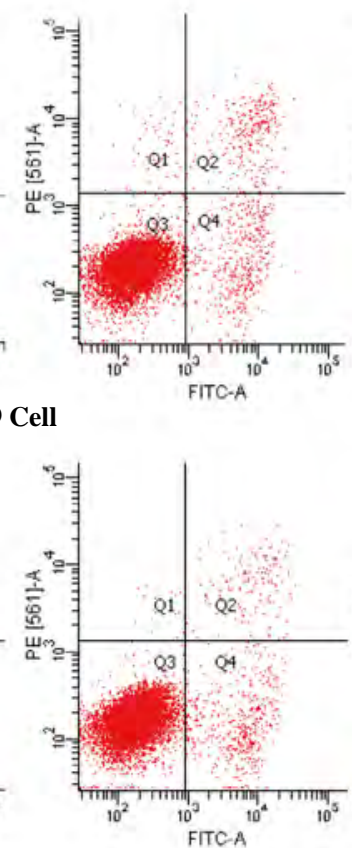

AC
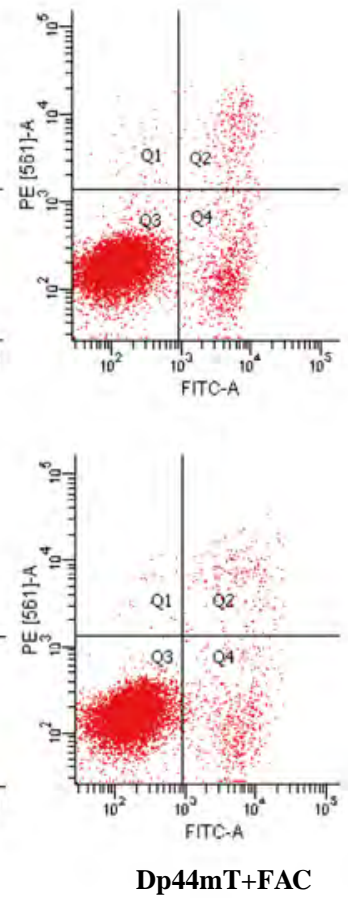

C

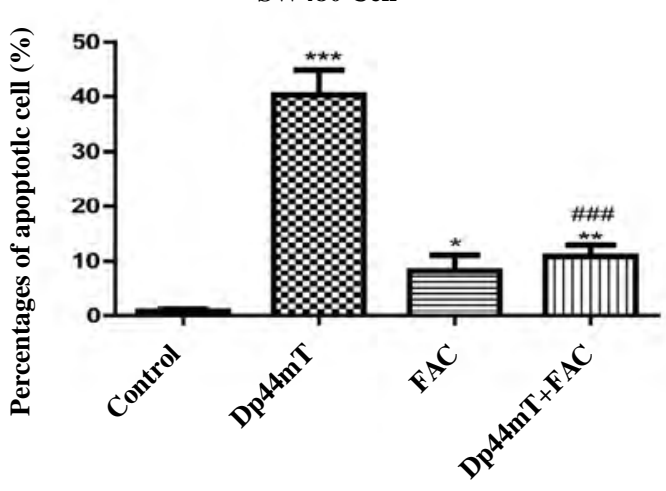

D

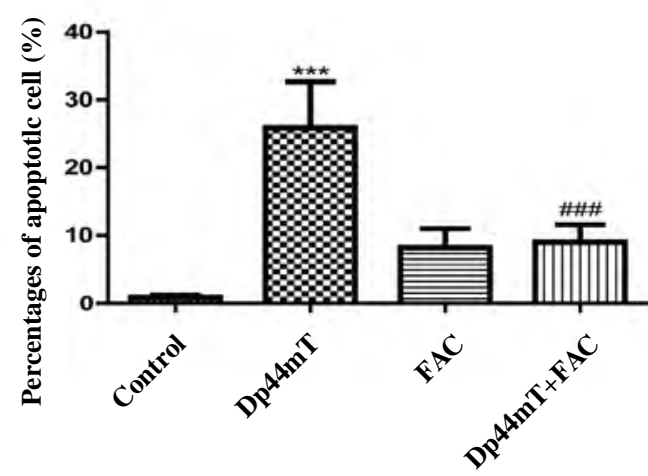

Figure 3. DFAC inhibited Dp44mT-induced SW480 and HT-29 cell apoptosis. Cells were stainedwith annexin V-FITC/PI to verify the apoptotic cell ratio. FAC inhibited Dp44mT-induced SW480 (A) and HT-29 (B) cell apoptosis. Percentages of apoptotic cells in the untreated control group, Dp44mT group $(10 \mu \mathrm{M})$, FAC group $(100 \mathrm{mg} / \mathrm{ml})$, and Dp44mT + FAC group were compared. Statistical results are shown in (C) and (D). ${ }^{*},{ }^{* *}$, and ${ }^{* * *}$ mean $P<0.05, P<0.01$ and $P<0.001$ compared with the control group, respectively. ${ }^{\# \#}$ means $P<0.001$ compared with the Dp44mT group; $n=3$.

DNA damage. The PI3K/AKT/mTOR pathway plays a critical role in the growth and progression of colorectal cancer. As the downstream protein of PI3K/Akt pathway, mTOR plays an important role in cell proliferation and apoptosis. In our study, we investigated the influence of Dp44mT on the phosphorylation level of mTOR and found that Dp44mT could significantily inhibit its activation. This founding may partialy explain the antitumor effects of Dp44mT and related iron chelators.

In conclusion, we explored the anti-tumor effects of the iron chelator Dp44mT on colorectal tumor cells using various assays. We found that Dp44mT could promote tumor cell apoptosis upregulate the expression level of pHistone H2A.X, and inhibit the phosphorylation level of mTOR in a dose-dependent way. These data indicate that iron depletion could modulate the human colorectal carci- noma (HCC) apoptosis progression in vitro, which may be a potential target for future HCC therapy.

\section{Declarations}

Authors' contributions: Qianqian Fu, Shengli Wang and Chuyun Zhu performed the experiments. Shengli Wang and Zhenlong Zhou analyzed the data. Qianqian Fu and Shengli Wang wrote the paper. Yong Zhang and Xiaojuan Yu were responsible for study supervision. All authors approved the final manuscript.

Availability of data and materials: Not applicable.

Financial support and sponsorship: None. 


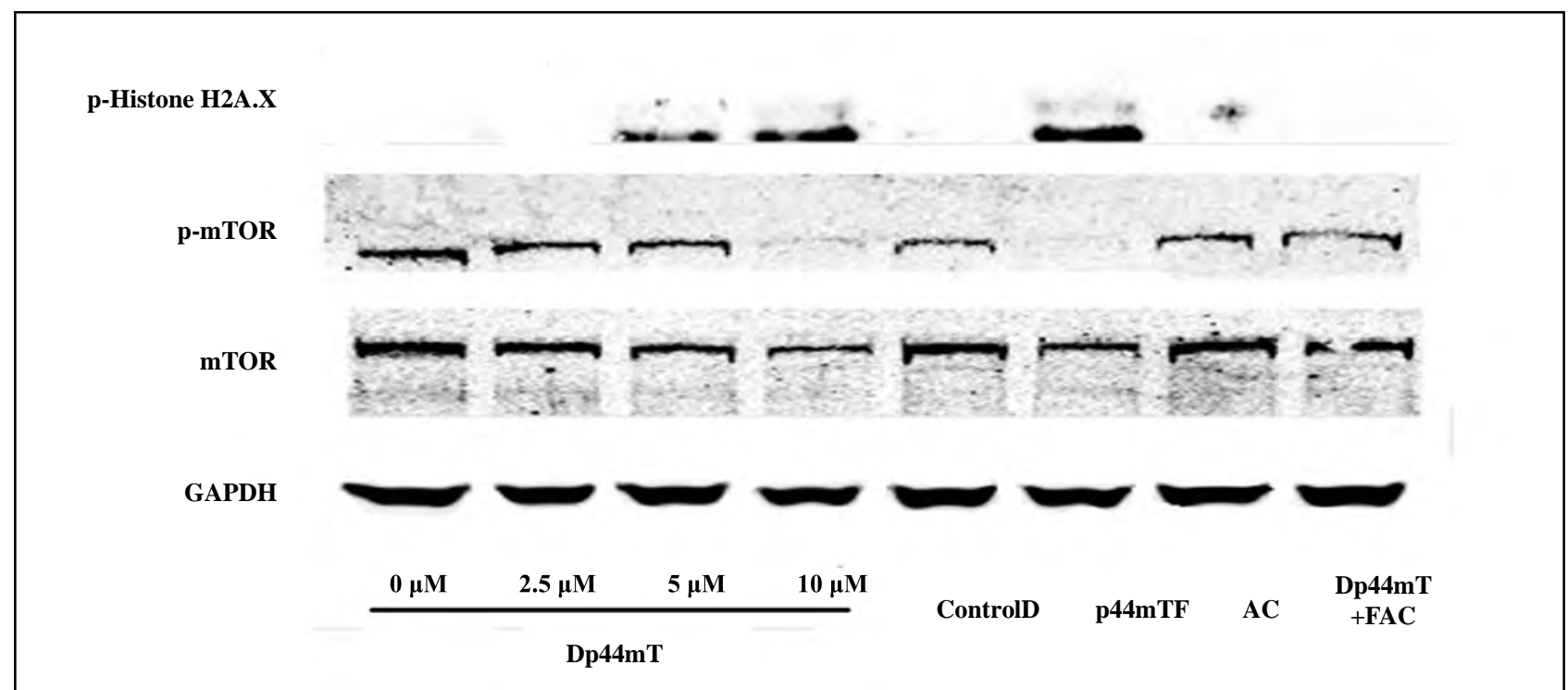

Figure 4. Dp44mT induced SW480 and HT-29 cell apoptosis via the mTOR pathway. Effects of different concentrations of Dp44mT $(0,2.5,5$, and $10 \mu \mathrm{M}$ ) and FAC on the protein expressions of p-Histone H2A.X, mTOR, and p-mTOR in SW480 cells. Dp44mT induced the protein expressions of p-Histone H2A.X and inhibited the phosphorylation of mTOR in a dose-dependent way. FAC inhibited Dp44mT-induced p-Histone H2A.X expression and recovered the phosphorylation of mTOR in SW480 cells.

Conflicts of interest: All authors declare that there are no conflicts of interest.

Ethical approval and consent to participate: Not applicable.

\section{References}

1. Brody H. Colorectal cancer. Nature, 2015, 521(7551): S1.

2. Gottesman MM, Fojo T, Bates SE, et al. Multidrug resistance in cancer: role of ATP-dependent transporters. Nat Rev Cancer, 2002, 2(1): 48-58.

3. Chaston T B, Lovejoy D B, Watts R N, et al. Examination of the antiproliferative activity of iron chelators: multiple cellular targets and the different mechanism of action of triapine compared with desferrioxamine and the potent pyridoxal isonicotinoyl hydrazone analogue 311 . Clinical cancer research, 2003, 9(1): 402-414.

4. Le N TV, Richardson D R. The role of iron in cell cycle progression and the proliferation of neoplastic cells. Biochimica Et Biophysica Acta-reviews On Cancer, 2002, 1603(1): 31-46.

5. Lovejoy D B, Jansson P J, Brunk U T, et al. Antitumor Activity of Metal-Chelating Compound Dp44mT Is Mediated by Formation of a Redox-Active Copper Complex That Accumulates in Lysosomes. Cancer research, 2011, 71(17): 5871-5880.

6. Yuan J, Lovejoy D B, Richardson D R. Novel di-2-pyridylderived iron chelators with marked and selective antitumor activity: in vitro and in vivo assessment. Blood, 2004, 104(5): 1450-1458.

7. Whitnall M, Howard J, Ponka P, et al. A class of iron chelators with a wide spectrum of potent antitumor activity that overcomes resistance to chemotherapeutics. Proceedings of the National Academy of Sciences of the United States of America, 2006, 103(40): 14901-1496.
8. Kovacevic Z, Chikhani S, Lovejoy D B, et al. Novel thiosemicarbazone iron chelators induce up-regulation and phosphorylation of the metastasis suppressor N-myc down-stream regulated gene 1: a new strategy for the treatment of pancreatic cancer. Molecular pharmacology, 2011, 80(4): 598-609.

9. Jansson P J, Yamagishi T, Arvind A, et al. Di-2-pyridylketone 4,4-dimethyl-3-thiosemicarbazone (Dp44mT) overcomes multidrug resistance by a novel mechanism involving the hijacking of lysosomal P-glycoprotein (Pgp). Journal Of Biological Chemistry, 2015, 290(15): 95889603.

10. Seebacher N A, Lane D J, Jansson P J, et al. Glucose Modulation Induces Lysosome Formation and Increases Lysosomotropic Drug Sequestration via the P-Glycoprotein Drug Transporter. Journal Of Biological Chemistry, 2016, 291(8): 3796-3820.

11. Kalinowski D S, Stefani C, Toyokuni S, et al. Redox cycling metals: Pedaling their roles in metabolism and their use in the development of novel therapeutics. Biochimica Et Biophysica Acta-molecular Cell Research, 2016, 1863(4): 727-748.

12. Yuan J, Lovejoy D B, Richardson D R. Novel di-2-pyridylderived iron chelators with marked and selective antitumor activity: in vitro and in vivo assessment. Blood, 2004, 104(5): 1450-1458.

13. Merlot A M, Shafie N H, Yu Y, et al. Mechanism of the induction of endoplasmic reticulum stress by the anti-cancer agent, di-2-pyridylketone 4,4-dimethyl-3-thiosemicarbazone (Dp44mT): Activation of PERK/eIF2 $\alpha$, IRE1 $\alpha$, ATF6 and calmodulin kinase. Biochemical Pharmacology, 2016, 109: 27-47.

14. Richardson D R, Sharpe P C, Lovejoy D B, et al. Dipyridyl thiosemicarbazone chelators with potent and selective antitumor activity form iron complexes with redox activity. Journal of medicinal chemistry, 2006, 49(22): 6510- 
6521.

15. Park J J, Lim K H, Baek K H. Annexin-1 regulated by HAUSP is essential for UV-induced damage response. Cell death \& disease, 2015, 6(2): e1654-e1654.

16. Guo Z L, Richardson D R, Kalinowski D S, et al. The novel thiosemicarbazone, di-2-pyridylketone 4-cyclohexyl4-methyl-3-thiosemicarbazone (DpC), inhibits neuroblastoma growth in vitro and in vivo via multiple mechanisms. Journal of hematology \& oncology, 2016, 9(1): 1-16.

17. Gutierrez E M, Seebacher N A, Arzuman L, et al. Lysosomal membrane stability plays a major role in the cytotoxic activity of the anti-proliferative agent, di-2-pyridylketone 4,4-dimethyl-3-thiosemicarbazone (Dp44mT). Biochimica Et Biophysica Acta, 2016, 1863(7): 16651681.
18. Seebacher N A, Richardson D R, Jansson P J. A mechanism for overcoming P-glycoprotein-mediated drug resistance: novel combination therapy that releases stored doxorubicin from lysosomes via lysosomal permeabilization using Dp44mT or DpC. Cell death \& disease, 2016, 7(12): e2510-e2510.

19. Wangpu X, Lu J, Xi R, et al. Targeting the Metastasis Suppressor, N-Myc Downstream Regulated Gene-1, with Novel Di-2-Pyridylketone Thiosemicarbazones: Suppression of Tumor Cell Migration and Cell-Collagen Adhesion by Inhibiting Focal Adhesion Kinase/Paxillin Signaling. Molecular pharmacology, 2016, 89(5): 521-540.

20. Greene B T, Thorburn J, Willingham M C, et al. Activation of caspase pathways during iron chelator-mediated apoptosis. Journal Of Biological Chemistry, 2002, 277(28): 25568-25575.

Cite this article as: Fu Q Q, Wang S L, Zhu C Y, et al. Di-2-pyridylketone 4, 4-dimethyl-3-thiosemicarbazone effectively induces human colorectal carcinoma cell apoptosis via mTOR pathway[J]. Aging Pathobiology and Therapeutics, 2021, 3(3): 56-62. 